\title{
Awareness Brazil - incidence of intraoperative awakening in a prospective study of I,259 cases
}

\author{
Volume I Issue 4 - 2014
}

\begin{abstract}
Background: Awareness anesthesia, during general anesthesia, has incidence quite variable in the literature. In high-risk patients, the incidence is $2 \%$, but in the general population the incidence varies between 0.1 to $0.2 \%$. In the USA, it is estimated that annually, are carried out around 21 million anesthesia's resulting in 20,000 to 40,000 new cases of peri-operative accidental awareness. In Brazil, there are no records, until nowadays.

Objective(s): This study primary evaluated the incidence of awareness anesthesia, considering only episodes declared explicit memory, in Brazil by using an electronic protocol for records. The secondary objectives were to compare the incidence of awareness anesthesia with inhalational general anesthesia/balanced (AI) versus total intravenous anesthesia (AVT) and evaluate the relationship of aspects related to the increase or the decrease of this incident, such as the use or non-use of neuromuscular blockers and benzodiazepines.
\end{abstract}

Design: Multicenter, prospective study through the application of an electronic questionnaire volunteer fill anesthetists and anonymous, now termed as: Awareness Brazil Protocol.

Setting: Ten thousand Anesthesiologists from around the country were invited to participate through access to an electronic address for filling in the questionnaire. Brazilian anesthesiologists were invited to participate through a formalized by e-mail invitation. In this invitation, if the colleague did was inserted a link to access an electronic page that contained the questionnaire. Public and private hospitals were invited to participate. The study was carried out during 5 months in the year 2013 .

Patients: The calculation of the sample was held establishing a representative sample among the anesthetic procedures performed in Brazil, taking into account the estimated number of procedures performed each year in the country $(15,000,000$ procedures for year), the maximum frequency of accidental awakening described in world literature $2 \%$ (due to lack of data on Brazilian literature), settling 2 degrees of freedom and $99 \%$ reliability, by adding $25 \%$ by way of errors and loss of data. The set number was 1,200 records needed in the sample with role-play collection. The technology of role-play collection was a collection tool used in order to make all participants collected the data the same way. 1,259 cases were collected corresponding to a confidence interval of $99 \%$. Exclusion criteria were refusal of the patient, patient's inability to participate within 24 hours after surgery and pediatric patients. Interventions: We research the presence of explicit memory after general anesthesia within a period of up to 24 hours after the realization of anesthesia. We compared the incidence of peri-operative accidental awareness with inhalational general anesthesia/ balanced (AI) versus total intravenous anesthesia (AVT) and evaluate the relationship of aspects related to the increase or the decrease of this incident, such as the use or non-use of neuromuscular blockers and benzodiazepines.

Main outcome measures: Explicit memory after general anesthesia was measured. Aspects that influence the occurrence of explicit memory, such as usage or not of muscle relaxants, benzodiazepines and type of anesthesia (balanced versus total intravenous) were analyzed.

Results: 1259 general anesthesia performed was registered throughout the country, during the data collection period. The incidence of peri-operative awareness evidenced by explicit memory was $2.5 \%(n=32)$. Statistical analysis of the incidences of awareness from general anesthesia performed with AVT and was significantly different. The incidence of awareness with AI was $3.50 \%(n=26)$ in a total of 737 cases performed with this technique. The AVT had an incidence of awareness of $1.14 \%(n=6)$ in a total of 522 registrations. Awareness anesthesia proved to be more frequent with the technique of AI compared to AVT

\author{
Diogenes Silva, Fernando Squeff Nora \\ Anestex Anesthesiology Clinic, Brazil
}

Correspondence: Diogenes Silva, São Francisco Hospital / Anestex Anesthesiology Clinic, Jeronimo Venancio das Chagas, 37 I apto 30I Condominio Atlantic Hall Campeche Florianopolis, SC Brazil,Tel 55-48-8833-1447, Email dr.diogenes@anestex.com.br

Received: August 20,2014 | Published: September 18, 2014

( $\mathrm{p}=0.0083 \mathrm{Mantel}-\mathrm{Haenszel}$ Chi-square). The relative risk of the patient awakening with AI was $56 \%$ higher when compared to AVT. General anesthesia was performed with the use of neuromuscular blockers $67.8 \%$ of the time $(n=854)$ and in the remaining $32.2 \%(n=405)$ the anesthesiologist have opted to not use neuromuscular blocker. In the group where awareness occurred peri-operative $(n=32), 81.5 \%(n=26)$ of the patients were under effect of neuromuscular blocker. The incidence of awareness when BZD was used during anesthetic induction, was of $28.13 \%(n=9)$ whereas when the BZD was not used the incidence of awareness was $71.88 \%(n=23)(p=0.438)$. The probability to be awakened when the BZD was not used was 2.8 times higher, compared to the group which used BZD. Of patients receiving BZD during induction and had awareness, $33.33 \%(n=3)$ were anesthetized with the technique of AVT and $66.67 \%(n=6)$ were anesthetized with AI. With a relative risk $>1(R R=1.4)$, concluded that the BZD lost protective action in greater intensity when general anesthesia technique used was inhalation/balanced. The incidence of awareness with pain was $25 \%(n=8)$ and without pain was $75 \%(n=24)$. The BIS or entropy soared in just $6.25 \%(n=2)$ of cases of awareness and in none of the cases where arousal and where there was suspicion of awareness by the Anesthesiologist. Conclusion: Under the conditions of this study, we conclude that: the incidence of awareness anesthesia evidenced by explicit memory, in Brazil is high when compared to global incidence, the AVT had lower incidence of awakening when compared to AI, movements and heart rate increase were more prevalent in the awareness of the suspicion that the elevation of the BIS, the incidence of awareness anesthesia was more frequent when there was use of neuromuscular blockers and decreased when BZD were used during induction (when the option of General anesthesia was by AVT mode). Additional studies need to be performed using validated alarm evaluation questionnaires in the literature as a specific group of Anesthesiologists committed to study in order to reduce the chance of records only when there is incident.

Trial registration: This protocol was redistricted at Brazil Platform (Health Government agency) and approved by National Health Council and National Commission of ethics in research-CONEP.

Keywords: Awareness, Anesthesia, Intravenous anesthesia, Inhalation anesthesia, Explicit memory 


\section{Introduction}

Awareness anesthesia, with explicit memory during general anesthesia, has an impact on world literature quite variable. These effects depend on many factors and may be related to anesthetic technique employed, the characteristics of patients and the nature of the operative procedure performed. The data collection form and the difficulty in creating more homogeneous groups of patients have generated quite disparate impact and incidences of peri-operative accidental awareness in literature. The majority of studies includes high-risk patients and compares the frequency of awareness in groups where the Bispectral index monitors are used and another group where these monitors are not used. Patients considered high risk raise this statistic for indexes around even $2 \%$, but in the general population the incidence of peri-operative awareness described with explicit memory ranges from 0.1 to $0.2 \% .^{1-15}$

This incidence may seem low. But, in the U.S.A, the Sentinel event number 32, published in 2004, described that are held annually, around 21 million anesthetics which, taking into account the epidemiological data currently described ( 0.1 to $0.2 \%$ ) result in 20,000 to 40,000 new cases of peri-operative accidental awareness with explicit memory, only in the territory of U.S.A., ${ }^{1,2}$

If we consider an estimated 15 million anesthetics each year held in Brazil, with an incidence of $2 \%$, we would arrive at an alarming number around 300 mil new cases of awareness a year. Here, we don't have this number or the total number of general anesthesia performed in the country, neither the incidence of awareness anesthesia with explicit memory, after general anesthesia. Several authors have conducted multicenter studies in order to determine the incidence, risk factors and the most appropriate forms of prevention and treatment of this incident that causes devastating psychological sequelae. Memories of hearing and feeling of not be breathing are the most frequent demonstrations and occur in $48 \%$ of cases of peri-operative accidental awareness anesthesia. Twenty-eight percent of patients report pain and $50 \%$ of them develop post traumatic stress syndrome, a framework that ranges from the development from the surgery of repetitive nightmares, anxiety related to sounds and/or images, panic and important behavioral changes that have taken these patients the need for specialized medical follow-up and treatment. In Brazil, we don't have sufficient data to evaluate the general incidence of peri-operative accidental awareness with presence of explicit memory. The primary objective of this study was to evaluate the incidence of awareness anesthesia, considering only episodes declared explicit memory, in Brazil, by applying an electronic notification called Awareness Brazil protocol (Appendix 1).

Explicit memory will be regarded, for the purposes of this study, like the one where the patient referred to in memory of events per-operative spontaneously to awareness or when asked if had some sort of reminder that he, patient, assign to the peri-operative period. ${ }^{5}$ The secondary objectives were to compare the incidence of peri-operative accidental awareness anesthesia with inhalational general anesthesia/ balanced (AI) versus total intravenous anesthesia (AVT) and evaluate the relationship of aspects related to the increase or the decrease of this incident, such as the use or non-use of neuromuscular blockers and benzodiazepines. Is not part of this study the determination or stratification of doses or concentrations of anesthetics used? While theoretically the doses of anesthetics used can influence the occurrence of awareness, in cases where there is the presence of awareness anesthesia the literature has analyzed only the presence or absence of drug classes where the occurrence of awareness would be increased or not. A more thorough analysis on the relationship of the doses used and the occurrence of awareness anesthesia will be made in the discussion.

\section{Methods}

We conducted a prospective, multicenter study through the application of an electronic questionnaire volunteer fill and anonymous, now termed as: Awareness Brazil protocol. For this was created a protocol in the form of electronic form where Brazil anesthesiologists professionals were invited to compute all data general anesthesia performed under his supervision. For the purposes of this Protocol was evaluated only the occurrence of accidental awareness with explicit memory determined for the spontaneously reminder of the patient to report situations experienced during general anesthesia. Ten thousand Anesthesiologists from around the country were invited to participate through access to an electronic address for filling out the questionnaire (Appendix 1).

In this invitation, if the colleague did was inserted a link to access an electronic page that contained the questionnaire (Appendix 2).

As we had no concrete data on the number of general anesthesia performed in Brazil, where it was held a general anesthesia, a record was held to generate a sample and, in case of occurrence of awareness, the colleague would be invited to fill in the fields for the occurrence of this incident. Colleagues who have agreed to participate in the study were instructed to ask the patient, within a period of up to 24 hours after the operation, if the patient had any memories that could be identified as occurring during the surgery, except dreams. Only cases of explicit memory were computed. Such memories could be auditory, visual or tactile. Patients who reported awareness anesthesia were asked if they had pain or not. The questionnaire was anonymous, voluntary and electronic. In a field appropriate to the Protocol described, had a specific protocol for the collection of data regarding the peri-operative accidental awareness anesthesia, characterized by memory of aspects related to surgery, if this item was marked. The electronic form recorded the data in the cloud, in a remote internet server different from that hosted the questionnaire for security measures, encrypting them and providing partial results in real time only search coordinators. The questionnaire used dynamic server pages technology optimized for mobiles (web app), allowing this to be accessed both computers as cell phones and tablets to ease data entry. The data were recorded in a database SQL (Structure Query Language), and subsequently analyzed in the software for epidemiological analysis, Epi-Info 6.04 and 7.1 releases. For statistical analysis of the data and the relationship between the variables, risks and probabilities, was used the Chi-square test of Mantel-Haenszel (CHM). Was considered statistically significant a $p<0.05$. The calculation of the sample was held establishing a representative sample among the anesthetic procedures performed in Brazil, taking into account the estimated number of procedures performed each year in the country, the maximum frequency of accidental awareness described in world literature $2 \%$ (due to lack of data on Brazilian literature), settling 2 degrees of freedom and $99 \%$ reliability, by adding $25 \%$ by way of errors and loss of data. From the set number of 1,200 records needed in the sample we use an e-form for data collection with facilitation role-play. The technology of role-play collection was a collection tool used in order to make all participants collected the data the same way. The data collection period was 5 months were 1,259 cases were collected corresponding to a confidence interval of $99 \%$. It is estimated that are carried out in Brazil around 15 million anesthetics procedures each year. How were evaluated only general anesthesia, it is possible that the estimated number of general anesthetics, per year in Brazil, is even smaller, which increases the chance that this data sample represents, with con- 
fidence index above $99 \%$, the Brazilian reality about the incidence of the event. This Protocol does not use paper or ink forms, respecting the precepts paperless for research. This research has obtained consent from the Research Ethics Committee of the hospital São Francisco de Assis. This protocol was registrated at Brazil Platform (Health Government agency) and approved by National Health Council and National Commission of ethics in research-CONEP.

\section{Results}

1259 general anesthesia performed was registered throughout the country, during the data collection period. The incidence of peri-operative awareness anesthesia was $2.5 \%(\mathrm{n}=32) .568$ patients were evaluated $(45.1 \%)$ male and female $691(54.9 \%)$. The average age of patients was $42,34 \mathrm{y}$. Anaesthesia performed on private network corresponded to $80.1 \%(\mathrm{n}=1008)$ of records and on the public network were $19.9 \%(n=251)$. The frequencies of surgical specialties involved in the study are listed in Table 1 , but General surgeries were the most frequently recorded with 508 cases $(40.4 \%)$. The operative time more often registered was up operations of $2 \mathrm{hs}$ duration $(\mathrm{n}=232)$ and Table 2 shows the records of all time. As for the character of the operation, $91.9 \%(n=1157)$ of operations occurred in elective and $8.1 \%(n=102)$ on the basis of urgency. Inhalation anesthesia or balanced (AI) was the technique of choice in $58.5 \%$ of cases $(n=737)$ and total intravenous anesthesia (AVT) was used in the remaining $41.5 \%(n=522)$.

Table I Kinds of surgical and frequencies

\begin{tabular}{lll}
\hline Surgical & n & $\%$ \\
\hline General & 508 & 40,4 \\
ORT & 178 & 14,1 \\
Orthopedics & 123 & 9,8 \\
Pasticsurgeries & 115 & 9,1 \\
Cardiac Sureries & 99 & 7,9 \\
Gynecology & 76 & 6,0 \\
Head and Neck & 65 & 5,2 \\
Oncologic Surgeries & 23 & 1,8 \\
Urology & 23 & 1,8 \\
Vascular & 22 & 1,7 \\
Trauma & 15 & 1,2 \\
Chest Surgeries & 8 & 0,6 \\
Obstetric Surgeries & 2 & 0,2 \\
Oftalmology Surgeries & 2 & 0,2 \\
\hline
\end{tabular}

Table 2 Durations of procedures

\begin{tabular}{lll}
\hline Intervals & $\mathbf{n}$ & $\%$ \\
\hline Until I hour & 232 & 18,4 \\
I to $2 \mathrm{hs}$ & 580 & $46, \mathrm{I}$ \\
2 to $3 \mathrm{hs}$ & 218 & 17,3 \\
3 to $4 \mathrm{hs}$ & 120 & 9,5 \\
Over $4 \mathrm{hs}$ & 109 & 8,7 \\
\hline
\end{tabular}

Statistical analysis of the incidences of awareness anesthesia from general anesthesia performed with AVT and AI were significantly different. The incidence of awareness anesthesia with AI was 3.50\% $(n=26)$ in a total of 737 cases performed with this technique. The AVT had an incidence of awareness anesthesia of $1.14 \%(n=6)$ in a total of 522 cases. Peri-operative awareness proved to be more frequent with the technique of AI compared to AVT ( $\mathrm{p}=0.0083$ Mantel-Haenszel Chi-square). The relative risk of the patient awareness anesthesia with AI was $56 \%$ higher when compared to AVT. General anesthesia was performed with the use of neuromuscular blockers $67.8 \%$ of the time $(n=854)$ and in the remaining $32.2 \%(n=405)$ the anesthesiologist have opted to not use neuromuscular blocker. In the group where awareness anesthesia occurred $(n=32), 81.5 \%(n=26)$ of the patients were under effect of neuromuscular blocker. The use of benzodiazepines (BZD) during anesthetic induction, occurred at $34.6 \%(n=435)$ of the cases and no BZD was used at $65.4 \%(\mathrm{n}=824)$. The incidence of awareness anesthesia when BZD was used during anesthetic induction, was of $28.13 \%(n=9)$ and whereas when the BZD was not used the incidence of awareness anesthesia was $71.88 \%(\mathrm{n}=23)(\mathrm{p}=0.438)$. The probability to be awakened when the BZD was not used was 2.8 times higher, compared to the group which used BZD. Of patients receiving BZD during induction and had awaken, $33.33 \%(n=3)$ were anesthetized with the technique of AVT and $66.67 \%(n=6)$ were anesthetized with AI. With a relative risk $>1(\mathrm{RR}=1.4)$, concluded that the BZD lost protective action in greater intensity when general anesthesia technique used was inhalation/balanced. The frequency of use of monitoring equipment as well as equipment related to the administration of anesthesia, such as vaporizers and target controlled infusion pumps, are described in Table 3. Among the $32(2.5 \%)$ patients reported awareness anesthesia, 23 (71.9\%) of them reported spontaneously the episode of memories during the operation, up to $8 \mathrm{hs}$ after the operation. Others were encouraged to respond if they had any recollection or heard something during the operation and the report was carried out within the first 24 hours after the operation. The incidence of awareness with pain was $25 \%(n=8)$ and without pain was $75 \%(n=24)$. The incidence of awareness when the anesthesiologist reported that suspected that the incident might have happened was $15.65 \%(\mathrm{n}=5)$. In the remaining $84.37 \%(n=27)$ of cases where the awareness anesthesia has occurred anesthesiologist reported not having suspicions that the incident could have occurred. The heart rate (HR) increased by $37.5 \%(n=12)$ of cases where arousal and in $80 \%(n=4)$ of those in which the anesthesiologist suspected of awareness anesthesia. The BIS or entropy soared in just $6.25 \%(\mathrm{n}=2)$ of cases of awareness and in none of the cases where arousal and where there was suspicion of awareness by the Anesthesiologist.

Table 3 Incidence of the use of monitoring equipment

\begin{tabular}{lll}
\hline Monitor/Monitoring Equipment & n & $\%$ \\
\hline Non Invasive Pressure & 1230 & 97,7 \\
Eletrocardiogram(EKG) & 1233 & 97,9 \\
Pulse Oximeter & 1222 & 97,1 \\
Intravenous Infusion Pump with Target Control & 555 & 44,1 \\
Gas Analyzer & 435 & 34,6 \\
Bispectral Index & 429 & 34,1 \\
Train of Four Monitor & 162 & 12,9 \\
Entropy & 3 & 0,2 \\
\hline
\end{tabular}

\section{Discussion}

We start this study describing an interesting introduction of a study entitled "Consciousness and Anesthesia - An update for the clinician, Teig et al. ${ }^{1}$ Let's say you are, at 7 o'clock of a Thursday morning entering the operating room of the hospital where he works. In front of you is the first patient of the day already you reports, at first contact, which is very anxious. After physical examination and history taking the patient asks: "How do you know if I am actually sleeping during the operation?" Experiences like these occur every day with all of us. And the answer isn't always easy. The first report of awareness anesthesia occurred in 1846, is as old as what we know about the anesthesia nowadays. ${ }^{1}$ In many situations, descriptions of patients with awareness anesthesia are very rich of details, since the narrative of conversations during the terrifying situations of complete operation until paralysis, pain and feeling of not be breathing. For this, the main factor related to awareness anesthesia configuration is the retention of memory or the memory of events per-operative after general anesthe- 
sia. A ratio around $50 \%$ of patients suffering from peri-operative accidental awareness during general anesthesia develop post trauma stress syndrome. This syndrome is characterized by night terrors, insomnia, nightmares, panic attacks related to sounds or even smell and is difficult to diagnosis and treatment. Involves the need for involvement of other professionals such as psychiatrists, besides being a major cause of litigation. Some patients develop unconscious panic related to hospital environments and others simply remember the experience throughout the surgery. Reports of patients who reported events of awareness anesthesia are diverse and with auditory memories (48\%), feeling of not be breathing (48\%) and pain (28\%). In the United States, are held annually, 21 million anesthesia resulting in an occurrence of awareness anesthesia, in absolute numbers, from 20,000 to 40,000 new cases a year. This means that arise each day 55 to 110 new cases of awareness anesthesia. ${ }^{2}$

1. Type of operation: Obstetrics, cardiac surgery and trauma, are among the most frequent.

2. Related to patients: Patients with a previous history of awareness anesthesia, in chronic use of benzodiazepines and/or opioids, with a previous history of drug addiction, physical status ASA IV, V and patients where provision has not been made for intubation difficulty due to the increased number of intubation attempts during induction of anesthesia.

3. Type of anesthesia: General anesthesia with the use of muscle relaxants, total intravenous anesthesia and lack of use of per-operative consciousness monitors has been described by the literature as additional risk factors

4. Factors related to equipment used: Equipment malfunction or breakage of inhalational anesthetics or administration of intravenous anesthesia, such as infusion pumps or vaporizers well as disconnection of venous or inhalator lines responsible for keeping the interface between equipment and patient.

5. Factors related to drugs in use by patients: Use of beta blockers can cause difficulty in responding increase of cardiac frequency during awareness anesthesia due to blockage of increased heart rate.

Similarly the use of calcium channel blockers, for the same reasons. Once characteristic signs and symptoms of awareness anesthesia, such as movements, increased blood pressure and heart rate, sweating, position of eyes and pupil reflexes are often and in many clinical situations masked or even impossible to be evaluated properly as that occurs, for example, during head and neck surgeries, where the anesthesiologist does not have access to the examination of the eyes of patients. This makes the little clinic assessment inaccurate. For the aspects related to incidence of awareness anesthesia in the general population, in this study we obtained an incidence of awareness anesthesia of $2.5 \%$, which, compared to world series $(0.1$ to $0.2 \%)$ was extremely high, even when we consider high-risk patients whose global incidence may reach up to $2 \% .^{5}$

In this study we did not controlled the risk patients and this could have caused a larger number of patients at risk of awareness anesthesia have been valued inadvertently raising the incidence. Several authors have described results quite varied as the incidence of awareness anesthesia. The difficulty in gathering data as well as the lack of a clear and objective classification makes the evaluation criteria and determination of re-awareness anesthesia are different. ${ }^{1-5}$ The definition that we used in our study was described by ASA and Teig et al. ${ }^{1,2-5}$ They described that awareness anesthesia is characterized by the ability of the patient to remember and related events that occurred during the peri-operative operation. Determined though so that the patient can report the event memory retention is required which can be divided into 2 types: implicit memory - that where the patient doesn't remember spontaneously from a peri-operative event, but through research techniques or use of specific questionnaires can remind of these events and, explicit memory when the patient is able to recount memories of peri-operative event spontaneously. The type of questionnaire and the form of collection of the data used in this study, without evaluating the incidence with a validated questionnaire specific search for awareness, may have raised this higher incidence since the participation in the study was voluntary and open to any anesthesiologist who remembered or had personal availability record the data. Thus, it is possible that this is done with that colleagues have registered more cases of awareness because when they had the incident resembled the study and recorded. This may have been an important bias of this study. The use of a specific questionnaire, as well as data collection in a more controlled may confirm or not this finding in a later study. Other authors reported incidences of up to 1 and $2 \%$ in patients considered high risk. ${ }^{6}$ On the other hand, a study of Sebel et al. ${ }^{7}$ found incidences as low as $0.13 \%$. Two other studies have found smaller incidences and between $0.023 \%$ and $0.0068 .^{8,9}$ Similarly, another study published recently that held an electronic questionnaire as ours, described an incidence of $0.028 \%$ in 85,156 anesthesia recorded by 172 anesthesiologists participants of the study. ${ }^{10}$ A second study, using the same questionnaire is being prepared to start another similar research, but using a questionnaire validated and with a specific group of guests to participate in the study in order to compare the results obtained here. When compared anesthetic techniques used, the results of this study were different than describes most studies surveyed in the world literature. While the world literature points to the AVT as a risk factor of awareness anesthesia, the analysis of the results of this study showed the opposite. When compared the cases of awakening, the AI presented 56\% more likely to be linked to awaken as compared to AVT. One aspect that might explain this finding is how the AVT has been held for the past 10 years with different modalities of administration and drugs. The vast majority of studies pointing to AVT as a risk factor for awareness anesthesia compiled older studies where the AVT was performed in such a way and with completely different medications of nowadays..$^{5-12}$

The use of target-controlled infusion equipment and the largest pharmacological knowledge by anesthesiologists, as well as more reliable medications are other reasons that can be pointed to the results reported here. Although it can be inferred that the AVT was safer by using target controlled infusion pumps, this hypothesis cannot be proven, in this study, statistically, requiring subsequent studies with even greater number of patients. This is the first study that showed that AVT had lower incidence of awareness anesthesia compared the AI with very forceful and significant results $(\mathrm{P}<0.0083)$. Important to mention that doses and concentrations of anesthetics were not reported here because this was not a study randomization factor. Our intent was to only compare the anesthetic techniques as are used by different anesthesiologists in the country and differently. Just categorize if it was AI or AVT. As the literature has described that clinically used concentrations (between 0.6 and 0.7 CAM) of AI and AVT tend to have the same incidence of awareness anesthesia, we not recorded the doses used because it would be irrelevant. ${ }^{5-9}$ The probability to be awareness anesthesia without the use of BZD during induction was 2.8 times greater under the conditions of this study, which is corroborated by previous publications. This fact corroborates with what is recommended by the American Society of Anesthesiology in BZD during anesthetic induction, especially when there are risk factors. ${ }^{5}$ Again, the recommendation of the ASA referred to only using BZD, but not mentions which the dose should be used and even the type 
of BZD, so we weren't able to determine these factors. Curious that, of patients receiving BZD during induction and had awaken, 33.33\% $(n=3)$ were anesthetized with the technique of AVT and others, $66.67 \%(n=6)$ were anesthetized with the technique of AI. This means that, in this study, it was possible to conclude that the BZD lost the protective action in greater intensity when the technique used was the inhalation/balanced (AI). With a relative risk $>1(\mathrm{RR}=1.4)$. The doses and types of BZD used may have influenced this finding, but we couldn't evaluate because we don't collect these data's. Of the signs and symptoms of awareness anesthesia, movement was the most frequent of them and increasing the heart rate (HR) occurred in $37.5 \%$ $(n=12)$ of cases where there was awareness and in $80 \%(n=4)$ of those cases in which the anesthesiologist suspected of awareness anesthesia and this in fact occurred. In other words, although the literature come questioning the validity of autonomic changes, such as heart rate, how reliable parameter for determining adequate anesthetic plane, in this study, the increase in heart rate, in cases of occurrence of awareness and with suspicion of awareness was the parameter most often pointed to in the records of awareness. In $100 \%$ of cases where awareness anesthesia occurred there was suspicion the Anesthesiologist that this might have occurred because the patient moved. It is advisable to note that muscle and beta blockers can alter cardiac chronotropic response and cause this signal is lost in patients using this class of medications. Its stresses the importance of greater care in patients using beta blockers and muscle relaxants. Under the conditions of this study, it was not possible to say that the diagnosis of awareness anesthesia or the suspects may have been based only and exclusively by the movement of the patients during peri-operative, mainly because not all suspected cases were confirmed as awareness. The BIS or entropy soared in just $6.25 \%(n=2)$ of cases where awareness anesthesia occurred and in none of the cases where there were concomitant suspicion of arousal and awareness by the Anesthesiologist. The use of BIS has shown significant reduction in the incidence of awareness anesthesia in several studies, although not enough evidence of $100 \%$ protection. ${ }^{4-9}$ There are several studies worldwide emphasizing the importance of this theme and situating the scientific community regarding the incidence, risk factors and, from these data, generating recommendations and support the scientific community. ${ }^{10-15}$ In Brazil, we didn't have any data available on the incidence of awareness anesthesia. Many studies have shown decreased incidence of awareness with the use of BIS, but the main multicenter study on use of BIS conducted in the world, named B-aware, presents results quite condundents as the protection offered by the BIS, when used in patients of high risk. ${ }^{11,12}$ Yet the routine use of BIS still does not find support in world literature, except in patients at high risk or when the use of neuromuscular blockers is indicated. ${ }^{5}$ With the cases of awareness anesthesia with AI the main cause pointed to was the malfunction of the vaporizer $(15.63 \%)$ $(n=5)$. During general anesthesia with AVT, in cases where awareness anesthesia occurred, was not reported any case of malfunction of the infusion pumps, but awareness occurred in one case (3.13\%) due to the disconnection of the devices of intravenous infusion of drugs.

Points out that, even though the majority of surgeries performed on an elective basis $(91.9 \%)$ the incidence of awareness was high. This study sought to provide general information about the incidence of accidental awareness from general anesthesia in Brazil. The incidence was quite high compared to world literature. Several factors may have contributed to the elevation of this incidence and may have been limiting factors in our study. Among them the lack of categorization of physical status (ASA), the non-correlation between cases where awareness has occurred with doses of anesthetics used. We not correlate the doses of anesthetics used because we assume that all anesthetics were carried out within the accepted clinical parameters and recommended by the literature. But certainly very disparate alterations in doses of anesthetics, and choices, if it occurred at some point, could cause significant distortions in the results. The average time of surgical procedures was not more 2 hours and was not stratified the incidence of awareness versus operation time because the procedures were mostly considered short. The analysis of the limiting factors of this study can have caused this increased incidence or not. Additional studies, stratifying doses, physical condition and type of hospital institution can answer these questions. Additional studies are needed to determine if this finding so impactful and high operative accidental awareness in Brazil to represent our reality or not. This was the first study conducted in Brazil evaluating awareness anesthesia and is extremely important, despite the limitations found because it begins a series of other studies and seeks to introduce the importance of cerebral monitoring during general anesthesia.

Under the conditions of this study, we conclude that: the incidence of awareness anesthesia in Brazil can be high when compared to global incidence, the AVT had lower incidence of awareness when compared to AI, movements and heart rate increase were more prevalent during awake episodes of the suspicion that the elevation of the BIS, the incidence of awareness anesthesia was more frequent when there was use of neuromuscular blockers and decreased when BZD were used during induction (when the option of General anesthesia was by AVT mode). Additional studies need to be performed using validated alarm evaluation questionnaires in the literature as a specific group of Anesthesiologists committed to study in order to reduce the chance of records only when there is incident.

\section{Funding details}

None.

\section{Acknowledgments}

None.

\section{Conflicts of interests}

Authors declare that there is no conflict of interest.

\section{References}

1. Teig MK, Hudetz AG, Mashour GA. Consciousness and Anesthesia. Advances in Anesthesia. 2010;30(1):13-27.

2. http://www.jointcommission.org/

3. Ghoneim MM (2000) Awareness during anesthesia. Anesthesiology. 92(2):597.

4. Nunes RR, Porto VC, Miranda VT, de Andrade NQ, et al. Risk factor for intraoperative awareness. Rev Bras Anestesiol. 2012;62(3):365-374.

5. American Society of Anesthesiologists Task Force on Intraoperative Awareness. Practice advisory for intraoperative awareness and brain function monitoring:a report by the American Society of Anesthesiologists task force on intraoperative awareness. Anesthesiology. 2006;104(4):847-864.

6. Leslie K, Davidson AJ. Awareness during anesthesia:a problem without solutions? Minerva Anesthesiol. 2010;76(8):624-628.

7. Sebel PS, Bowdle TA, Ghoneim MM, et al. The Incidence of Awareness During Anesthesia:a Multicenter United States Study. Anesth Analg. 2004;99(3):833-839.

8. Pollard RJ, Coyle JP, Gilbert RL, et al. Intraoperative awareness in a regional medical system:a review of 3 years data. Anesthesiology. 2007;106(2):269-274. 
9. Mashour GA, Wang LY, Turner CR, et al. A retrospective study of intraoperative awareness with methodological implications. Anesth Analg. 2009;108(2):521-526.

10. Morimoto Y, Nogami Y, Harada K, et al. Awareness during anesthesia:the results of a questionnaire survey in Japan. J Anesth. 2011;25(1):72-77.

11. Myles PS, Leslie $\mathrm{K}$, McNeil J, et al. Bispectral index monitoring to prevent awareness during anaesthesia:the $\mathrm{b}$ - aware randomised controlled trial. Lancet. 2004;363(9423):1757-1763.

12. Ekman A, Lindholm ML, Lennmarken C, et al. Reduction in the incidence of awareness using BIS monitoring. Acta Anaesthesiol Scand. 2004;48(1):20-26.
13. Lennmarken C, Sandin R. Neuromonitoring for awareness during surgery. Lancet. 2004;363(9423):1747-1748.

14. Osterman JE, Hopper J, Heran WJ, et al. Awareness under anesthesia and the development of posttraumatic stress disorder. General Hospital Psychiatry. 2001;23(4):198-204.

15. Spitellie PH, Holmes MA, Domino KB. Awareness during anesthesia. Anesthesiol Clin North America. 2002;20(3):555-570. 